\title{
Bridging Cytoarchitectonics and Connectomics in Human Cerebral Cortex
}

\author{
Martijn P. van den Heuvel, ${ }^{1}$ Lianne H. Scholtens, ${ }^{1}$ Lisa Feldman Barrett, ${ }^{2,3}$ Claus C. Hilgetag, ${ }^{4}$ and Marcel A. de Reus ${ }^{1}$ \\ ${ }^{1}$ Brain Center Rudolf Magnus, Department of Psychiatry, University Medical Center Utrecht, 3508 GA Utrecht, The Netherlands, ${ }^{2}$ Department of \\ Psychology, Northeastern University, Boston, Massachusetts 02115, 32Department of Psychiatry and Athinoula A. Martinos Center for Biomedical Imaging, \\ Massachusetts General Hospital and Harvard Medical School, Boston, Massachusetts 02129, and ${ }^{4}$ Department of Computational Neuroscience, University \\ Medical Center Hamburg-Eppendorf, 20246 Hamburg, Germany
}

The rich variation in cytoarchitectonics of the human cortex is well known to play an important role in the differentiation of cortical information processing, with functional multimodal areas noted to display more branched, more spinous, and an overall more complex cytoarchitecture. In parallel, connectome studies have suggested that also the macroscale wiring profile of brain areas may have an important contribution in shaping neural processes; for example, multimodal areas have been noted to display an elaborate macroscale connectivity profile. However, how these two scales of brain connectivity are related-and perhaps interact-remains poorly understood. In this communication, we combined data from the detailed mappings of early twentieth century cytoarchitectonic pioneers Von Economo and Koskinas (1925) on the microscale cellular structure of the human cortex with data on macroscale connectome wiring as derived from high-resolution diffusion imaging data from the Human Connectome Project. In a cross-scale examination, we show evidence of a significant association between cytoarchitectonic features of human cortical organization-in particular the size of layer 3 neurons - and whole-brain corticocortical connectivity. Our findings suggest that aspects of microscale cytoarchitectonics and macroscale connectomics are related.

Key words: connectivity; connectomics; cytoarchitectonics; diffusion MRI; MRI; pyramidal neuron

\section{Significance Statement}

One of the most widely known and perhaps most fundamental properties of the human cortex is its rich variation in cytoarchitectonics. At the same time, neuroimaging studies have also revealed cortical areas to vary in their level of macroscale connectivity. Here, we provide evidence that aspects of local cytoarchitecture are associated with aspects of global macroscale connectivity, providing insight into the question of how the scales of micro-organization and macro-organization of the human cortex are related.

\section{Introduction}

The human brain comprises a complex network of neural connections known as the human connectome. At the microscale, neurons are linked by dendrites, axons, and synapses, ensuring

Received July 10, 2015; revised Aug. 5, 2015; accepted Aug. 17, 2015.

Author contributions: M.P.v.d.H. designed research; M.P.v.d.H. and L.H.S. performed research; M.P.v.d.H. and M.A.d.R. contributed unpublished reagents/analytic tools; M.P.v.d.H., L.H.S., and M.A.d.R. analyzed data; M.P.v.d.H., L.H.S., L.F.B., C.C.H., and M.A.d.R. wrote the paper.

Human neuroimaging data were kindly provided by the Washington University, University of Minnesota, and Oxford University (WU-Minn HCP consortium; Principal Investigators: David Van Essen and Kamil Ugurbil; Grant 1U54MH091657) funded by the 16 National Institutes of Health (NIH) institutes and centers that support the NIH Blueprint for Neuroscience Research and by the McDonnell Center for Systems Neuroscience at Washington University. M.P.v.d.H. is supported by the Netherlands Organization for Scientific Research (VENI Grant 451-12-001).

Correspondence should be addressed to Martijn van den Heuvel, Brain Center Rudolf Magnus, Department of Psychiatry, University Medical Center Utrecht, Heidelberglaan 100,3508 GA Utrecht, P0 Box 85500, Room: A01.126, The Netherlands. E-mail: M.P.vandenheuvel@umcutrecht.nl.

DOI:10.1523/JNEUROSCI.2630-15.2015

Copyright $\odot 2015$ the authors $\quad 0270-6474 / 15 / 3513943-06 \$ 15.00 / 0$ transmission, dissemination, and integration of neural information. In parallel, at the macroscale level of brain organization, cortical and subcortical regions are connected by long-distance white matter projections, enabling neural communication and integration of information across different parts of the brain. However, how these two scales of brain organization relate- or perhaps interact-remains poorly understood.

Evidently, organizational features at both the microscale and macroscale are of crucial importance to brain functioning. Work of neuroanatomists such as Brodmann (1909); Vogt and Vogt (1903), and Von Economo and Koskinas (1925) revealed a rich variety in cytoarchitecture of the human cortex and, expanding the thoughts of these early pioneers, contemporary neuroanatomy studies have suggested that cytoarchitectonic differentiation may play a fundamental role in the type of information processed by cortical areas (Sanides, 1970; Zilles et al., 2015a,2015b; Barbas, 2015). Additional support for such theories may come from stud- 
A

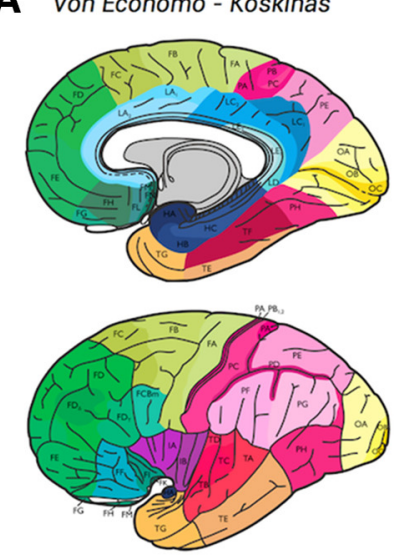

C
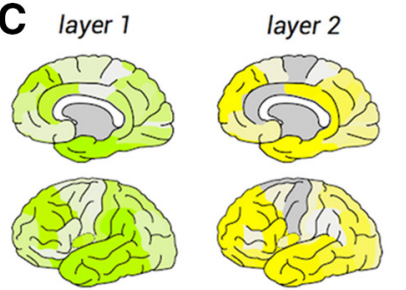

Desikan-Killiany 57 mapping
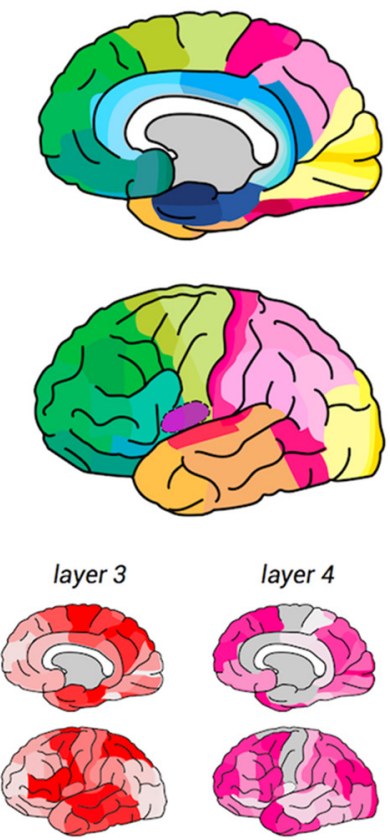
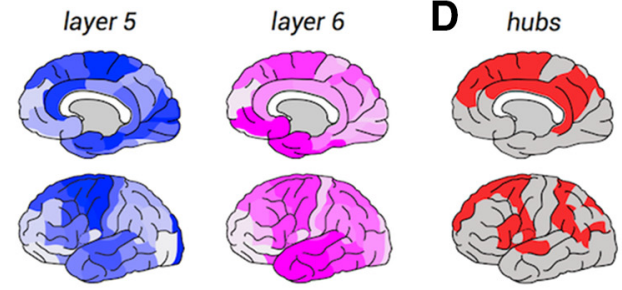

Figure 1. A, Digital version of the cytoarchitectonic Von Economo and Koskinas (1925) parcellation (left) and the mapped subregions of the Desikan-Killiany (DK-57) atlas (right). $\boldsymbol{B}$, Nodal strength of macroscale connectivity for the DK-57 regions (left hemisphere). C, Derived Von Economo-Koskinas values of neuron size for the six layers mapped on the DK-57 areas. D, Regions considered as connectome hubs based on their macroscale projections.

ies examining the organization of neurons by means of Golgi staining techniques showing that associative frontal areas of the human and macaque cortex (e.g., BA 46/10/11) display elaborate neuronal architectures with larger, more branched, and more spinous pyramidal neurons compared with unimodal primary regions (e.g., BA 17/41/42) (Jacobs et al., 2001; Elston, 2003; Yuste, 2011). Together, these observations have led to influential theories on regional differences in cytoarchitecture and the associated degree of laminar differentiation to play a pivotal role in shaping the dynamics of neural processing and corticocortical communication (Zilles et al., 2015a, 2015b; Barbas, 2015; Mesulam, 1998).

In parallel, studies examining connectivity at the macroscale level of brain organization have suggested that the brain's global network structure of large-scale white matter projections may play an important role in neural processing. For example, macroscale connectome studies have suggested large variation in the connectivity profiles of cortical regions, with some regions showing predominantly local short-range projections and other regions displaying more elaborate connectivity profiles suitable for sending and receiving neural information across different parts of the cortex. Somewhat analogous to the microscale, regional variation in macroscale connectivity profile has been directly related to the type of information processed by cortical regions, with densely wired "brain hubs" noted to show high spatial overlap with functional multimodal association areas and, accordingly, being thought to act as central integration zones of the cortex (for review, see van den Heuvel and Sporns, 2013).

While it is thus apparent that features of connectivity at both the microscale and macroscale play a pivotal role in brain functioning, it is much less clear how these two scales of neural connectivity are related. In this brief communication, we show evidence of a putative microscale-macroscale relationship across the human cortical mantle by combining data from the cortical mappings of cytoarchitectonic pioneers Von Economo and Koskinas (1925) with data on macroscale connectome organization as derived from high-resolution diffusion MRI data from the Human Connectome Project (HCP) (Van Essen et al., 2013).

\section{Materials and Methods}

\section{Von Economo and Koskinas cytoarchitectonic metrics}

Cytoarchitectonic mappings of the human cortex were taken from the 1925 Von Economo and Koskinas work Die Cytoarchitektonik der Hirnrinde des erwachsenen Menschen (Von Economo and Koskinas, 1925) [translated Cytoarchitectonics of the Adult Human Cerebral Cortex (Triarhou, 2008)]. As described in their accompanying 1925 writings and in Von Economo's 1927 writings, Zellaufbau der Grosshirnrinde des Menschen, based on a series of his teaching lectures (von Economo, 1927) and translated as Cellular Structure of the Human Cerebral Cortex (Triarhou, 2009), Von Economo and Koskinas described a parcellation of the whole human cortex into 48 "Grundareae" regions ("ground" areas), with a finer parcellation into 76 smaller "Varianten" ("variants"). For 51 regions ( 40 ground areas and 8 variants covering the whole cortex, together with three hippocampal areas), Von Economo and Koskinas listed detailed tables including layer specific information on (1) neuronal count per cubic millimeter, (2) layer width, and (3) neuron width and length defining neuron size (taken here as an ellipse multiplication of the reported cell width and length; Von Economo and Koskinas, 1925). The 48 cortical regions were manually mapped to a 57 -region subdivision of the FreeSurfer Desikan-Killiany atlas (describing 57 regions in each hemisphere; Fig. 1; Fischl et al., 2004; Hagmann et al., 2008) using the original 1925 and 1927 text and drawings. The drawings were overlaid and manually projected onto a $3 \mathrm{D}$ cortical surface reconstruction of the FreeSurfer average brain (Fischl et al., 2004) from which the overlap with the DK-57 atlas was determined. A complete textual and visual description of this mapping (together with a validation for the DK-57 atlas, as well as for atlases with other resolutions) was performed as part of a recent study in which we examined the validity of MRI T1-based estimates of cortical thickness by means of ground-truth histological examinations of cortical mantle width by Von Economo and Koskinas (Scholtens et al., 2015). Mapping resulted in a listing of cytoarchitectonic values for each of the 
A

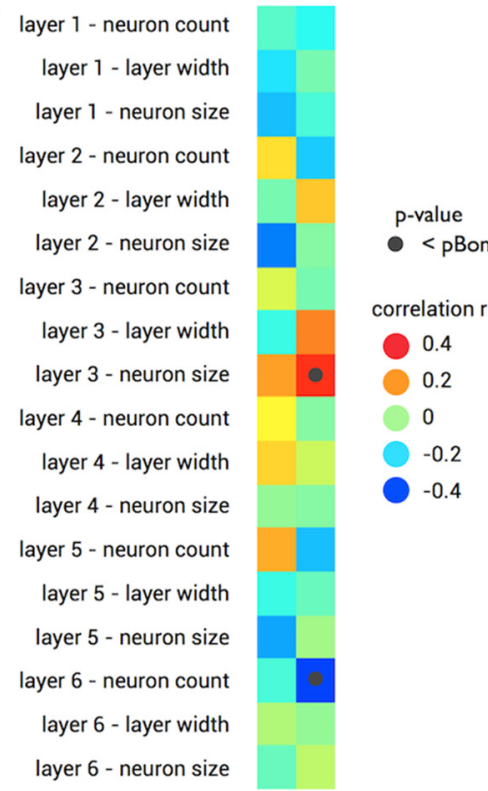

B

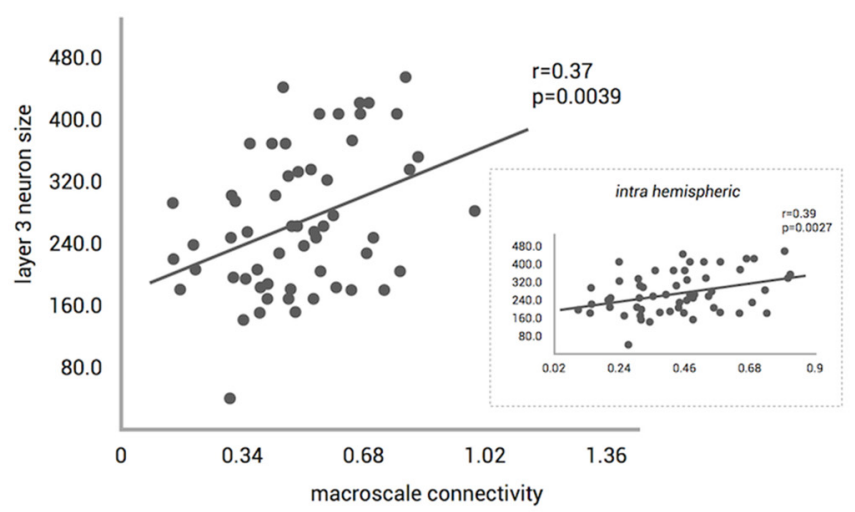

C

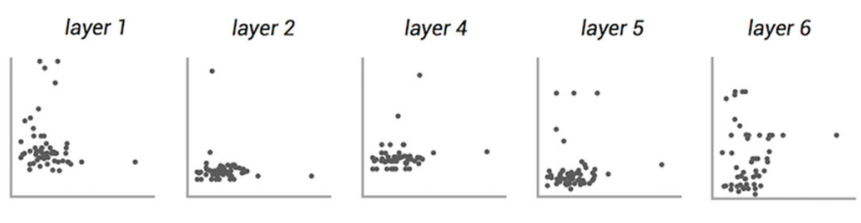

Figure 2. A, Correlation matrix between all 18 microscale cytoarchitectonic metrics (6 layers $\times 3$ metrics) and the two examined macroscale connectome values (degree and strength). $\boldsymbol{B}$, Positive association between layer 3 neuron size ( $y$-axis) and macroscale connectivity strength of cortical regions (i.e., nodal strength, $x$-axis). Insert shows the association between layer 3 neuron size ( $y$-axis) and intrahemispheric nodal strength ( $x$-axis, left hemisphere). C, Panels illustrate an absence of a micro-macro association for the other layers.

DK-57 regions, describing 18 [3 (neuron count, layer thickness, and neuron size) $\times 6$ (layers)] metrics of cytoarchitectonic organization for each cortical area.

Supragranular architecture. In addition to layer specific neuron count, the degree of supragranular differentiation of cortical regions was computed. The relative number of originating supragranular neurons (SGN\%) is often used as a metric of the degree of laminar differentiation and has been noted to form an important marker for the distribution of corticocortical projections (Hilgetag and Grant, 2010). Following the definition of Barbas and coworkers (Barbas and Rempel-Clower, 1997; Hilgetag and Grant, 2010), the degree of laminar differentiation SGN\% was computed as the balance between SGN and infragranular neurons (IGN) as SGN/(SGN + IGN), with SGN the number of neurons in supragranular layers 2 and 3, computed as the Von Economo and Koskinas reported neuron count per cubic millimeter multiplied by regional layer thickness and cortical surface area, and IGN the total number of neurons in infragranular layers 5 and 6 .

\section{Macroscale MRI connectome reconstruction and analysis}

Connectome reconstruction. High-resolution diffusion-weighted data from the HCP (Van Essen et al., 2013; Q3 release; $n=215$ subjects, males and females mixed, age 22-35 years; imaging parameters: voxel size 1.25 $\mathrm{mm}$ isotropic, TR/TE $5520 / 89.5 \mathrm{~ms}, 270$ diffusion directions with diffusion weighting 1000,2000 , or $3000 \mathrm{~s} / \mathrm{mm}^{2}$ ) was used to reconstruct a macroscale human connectome map. Diffusion weighted imaging data processing included the following: (1) eddy current and susceptibility distortion correction, (2) reconstruction of the voxelwise diffusion profile using generalized q-sampling imaging, and (3) whole-brain streamline tractography (for details on HCP connectome reconstruction, see also de Reus and van den Heuvel, 2014). Cortical segmentation and parcellation was performed on the basis of a high-resolution T1weighted image (voxel size: $0.7 \mathrm{~mm}$ isotropic) of each subject using FreeSurfer (Fischl et al., 2004), automatically parcellating the complete cortical sheet into 114 distinct regions (57 per hemisphere) using the DK-57 atlas (Cammoun et al., 2012). For each individual subject, a $114 \times$ 114 connectivity matrix was formed, representing for each pair of regions their reconstructed pathways. The strength of the reconstructed connections was measured using the notion of streamline density, computed as the number of tractography streamlines that touched both cortical regions divided by their average cortical surface area (Hagmann et al.,
2008). A group-level human connectome map was formed by taking the nonzero mean across the individual connectivity matrices, including connections that were observed in at least $60 \%$ of the subjects (van den Heuvel and Sporns, 2011).

Connectome analysis. With the connectome map describing a mathematical graph for each cortical region, the level of nodal degree was computed as the number of binary connections of a region and the level of nodal strength was computed as the total sum of connectivity strength across all reconstructed projections of a region. Because the Von Economo and Koskinas mappings did not distinguish between hemispheres, nodal connectivity properties of the left hemispheric regions were taken for analysis.

\section{Statistical analysis}

Associations between regional variation in cytoarchitectonic features and macroscale connectome properties were examined by means of Pearson correlation analysis. Correlations reaching a partial Bonferroni $\alpha$ of $0.0083[0.05 /(3 \times 2)]$ were taken as significant, corresponding to $3 \mathrm{mi}-$ croscale factors derived from a principal component analysis on the 18 micrometrics, with the 3 components describing $>99 \%$ of the variance and the 2 macrometrics (nodal degree and strength). For additional tests, a Bonferroni threshold of $0.05 / 4=0.0125$ was taken.

\section{Results}

Figure $2 A$ shows the full matrix of correlation values between the examined microscale and macroscale metrics. Layer 3 neuron size significantly correlated to the regional connectivity strength of macroscale connectome pathways $(r=0.37, p=0.0039$; Fig. $2 B$; effect reaching partial Bonferroni correction). Examining nodal strength across intrahemispheric connections (i.e., thus taking the strength of a node as the sum over its intrahemispheric corticocortical connections) revealed a similar positive association with layer 3 neuron size $(r=0.39, p=0.0027$; Fig. $2 B$, insert). Other layers (layer 1, 2, 4, 5, and 6) showed no clear link between nodal strength and neuron size (Fig. $2 C$, all $p>0.05$ ).

Looking into the layer 3 cortical association in more detail, no effect was found with total neuron count $(r=0.034, p=0.79$; Fig. $2 A$ ). Partial correlation of nodal connectivity strength with layer 
3 neuron size (regressing out the effects of neuron count by including it as a covariate) thus similarly resulted in a significant association between macroscale connectivity strength and layer 3 neuron size $(p=0.0040)$. Further regressing out the effect of the thickness of layer 3 (with layer 3 thickness and neuron size correlating, $r=0.32$ ) again revealed a relation (that was somewhat attenuated) between layer 3 neuron size and macroscale connectivity $(p=0.0186)$. In addition, a negative correlation was observed between layer 6 neuron count and regional macroscale connectivity ( $p=0.0027$; Fig. $2 A$ ), but this effect was attenuated when examining intrahemispheric connectivity only $(p=$ 0.0147).

\section{Laminar differentiation}

SGN\% was found to be significantly correlated to regional variation in macroscale connectivity strength $(r=0.38, p=0.0045$; Fig. 3$)$, with more densely connected cortical areas showing an overall more developed supragranular structure.

\section{Hubs, communities, and cytoarchitectural overlap}

Hub regions - selected as the top 20\% highest degree nodes in the network (van den Heuvel and Sporns, 2011)_included subregions of the caudal middle frontal cortex, inferior and superior parietal cortex, middle and superior temporal cortex, subparts of the inferior (parsopercularis) and superior frontal gyrus, precuneus, as well as the most inferior subpart of the precentral gyrus (Von Economo-Koskinas region FB/FBop, inferior part of Brodmann Area 6) and subparts of the supramarginal gyrus and insular cortex, regions highly consistent with those described previously (Hagmann et al., 2008; van den Heuvel and Sporns, 2011; Fig. 1D). This set of network hubs showed, on average, larger layer 3 neurons ( 1.29 times larger, $p=0.0112$, permutation testing, 10,000 permutations) compared with peripheral nodes (meaning low-degree regions). [Hub selection thresholds of $10 \%$ (1.33 $\times, p=0.0226$, not reaching Bonferroni correction) and $30 \%(1.25 \times, p=0.0122)$ revealed similar findings.]

Next, we examined regional involvement in connectome communities and overlap in the cytoarchitectonic profile of cortical regions. Between all pairs of cortical areas, region-to-region correspondence in the cytoarchitectonic profile was computed by correlating profiles of regions (before computing the correlation coefficients each microscale factor was normalized by dividing by the mean and redistributing the values to a Gaussian distribution). Cortical regions belonging to the same structural network community (module detection using Newman's modularity algorithm identified 5 anatomical communities) showed a significantly higher cytoarchitectural correspondence than region pairs belonging to communities ( 1.25 times higher, $p=0.0028$, permutation testing 10,000 permutations).

\section{Discussion}

Our findings provide evidence of a correlation between microscale cytoarchitectonics and macroscale connectomics of human cortex. We show that macroscale connectivity of cortical regions is not directly related to neuronal count and/or layer thickness of the cortical mantle [noted to correspond well to MRI-derived estimates of cortical thickness (Scholtens et al.,
2015)]. Rather, our findings show that the organization of macroscale projections correlates with cortical variation in neuron size, in particular the size of neurons in cortical layer 3. Furthermore, more developed supragranular structure of cortical areas was found to be associated to stronger corticocortical macroscale connectivity. Supragranular layers (and in particular layer 3) of the mammalian cortex are known to form a primary layer for long-range corticocortical communication (for overview, see Jacobs et al., 1997; Jacobs et al., 2001; Schuz, 2002; Barbas, 2015), supporting our observation that macroscale connectivity is related to microscale cytoarchitectonic properties.

The monumental-but perhaps by today's neuroscientists sometimes forgotten-1925 work of Von Economo and Koskinas is arguably one of the most detailed and most complete listings of cytoarchitectonic features of the human cortical mantle, but it is of course inherently limited by the technology and methodology of that time (for discussion, see Triarhou, 2009; Amunts and Zilles, 2012). The writings of Von Economo and Koskinas (1925) do not mention the exact number of samples, making the study power perhaps less than we are used to in modern day examinations. Von Economo and Koskinas nevertheless explicitly state that every region was consistently observed in all investigated samples, with recordings based on histological examinations of - as they emphasize- "zahlreichen Gehirnen" ("numerous brains"; Von Economo and Koskinas, 1925) of healthy subjects (i.e., no reported history of neurological and/or psychiatric disorders). Furthermore, it should be noted that the from the work of Von Economo and Koskinas (1925) derived metric of cell size- derived from their at that time newly adopted notation of "Schlankheit" (slenderness) of a neuron (Von Economo and Koskinas, 1925)_includes a simplified metric of neuronal organization compared with metrics provided by modern day $3 \mathrm{D}$ techniques that allow for complete reconstructions of neuron dendritic trees and detailed measurements of spine count, spine type, and so forth (Jacobs et al., 2001; Lichtman and Sanes, 2008). To provide some insight into this matter, we examined the value of layer 3 neuron size as an indicator for neuronal complexity in a post hoc analysis. Examining layer 3 pyramidal neuron size in a recently collated dataset on layer 3 neuronal and dendritic organization of cortical regions in the macaque cortex (Elston, 2003; Scholtens et al., 2014), we observed pyramidal 
soma size to be highly correlated to measurements of dendritic tree size $(r=0.67, p=0.0013)$, spine count $(r=0.71, p=$ 0.0004 ), and spine density (number of spines per unit of tree length, $r=0.72, p=0.0005$ ), suggesting that neuron soma size is a simplistic but nevertheless useful marker for neuronal organization.

With neurons most often interpreted as being biological accumulators and integrators of neural information, larger neuron size and accompanying higher complexity of dendritic branching has been related to a higher neural processing and integration capacity of cortical areas (Elston et al., 2001; Yuste, 2011). In their work on morphological aspects of pyramidal neurons in the macaque cortex, Elston and colleagues noted a gradient of pyramidal complexity from posterior to anterior regions, with association regions showing more elaborate pyramidal organization (Elston, 2003; Scholtens et al., 2014). From a functional perspective on brain processes, the "global workspace hypothesis" (Dehaene et al., 1998) describes the existence of a functional system in which information can be integrated through means of abundant communication and interaction, a system that is hypothesized as a distributed set of cortical regions with dense levels of mutual and bidirectional projections and, to be able to process high amounts of neuronal information, a system of multimodal regions with an elaborate cytoarchitectonic organization (Dehaene and Naccache, 2001). Studies have identified hub regions in the mammalian cortex to constitute a widespread anatomical "rich club" system with an above average level of mutual and mostly reciprocal interregional connectivity (van den Heuvel and Sporns, 2013) and, from this, we and others have hypothesized the neural rich club to form an ideal candidate for an anatomical substrate of a global workspace in the mammalian brain (for review, see van den Heuvel and Sporns, 2013). We argue that our current findings add another piece to this puzzle, confirming macroscale high-degree hub areas of the human cortex to indeed, as hypothesized, display an intricate neuronal organization on the microscale.

The notion of an association between microscale and macroscale properties of neural connectivity and complexity is consistent with recent observations from animal studies. Beul et al. (2014) reported that cytoarchitectonic classes of areas of cat cortex overlap with patterns of macroscale connectivity as derived from tract-tracing experiments. We previously reported the neural complexity (e.g., spine density, dendritic branching) of layer 3 pyramidal neurons of a subset of areas of macaque cortex to be related to macroscale connectivity of cortical regions (Scholtens et al., 2014). Extending such observations now to the whole human cortex, our findings converge on the notion of architectonic aspects of connectivity at the microscale and macroscale level of brain organization to be associated-and to possibly interact- to shape the flow of corticocortical communication (Barbas, 2015; van den Heuvel et al., 2012).

Our study shows encouraging findings of an association between microscale cytoarchitectonics and macroscale connectomics of the human cortex, but several aspects have to be taken into account when interpreting the results. It has to be noted that, in addition to limitations concerning the dimensionality of the Von Economo and Koskinas cytoarchitectonic mappings (see above), the reconstructions of macroscale connectome maps are also inherently limited by the techniques used. Even though the data of the HCP is of very high quality, diffusion tractography remains a technique that depends on indirect information (the hindered diffusion of water molecules by fiber structure) for the reconstruction of anatomical pathways. As a result, diffusion tractography is known to have limitations with respect to the reconstruction of white matter projections in areas with a complicated pathway layout and has been argued to involve both overrepresentations and underrepresentations of connectivity (Jbabdi and Johansen-Berg, 2011) compared with animal tract tracing-based connectome maps (Markov et al., 2013).

Our findings converge on the notion that aspects of the brain's smallest and largest organizational features of neural connectivity are associated (Oh et al., 2014). Studying the fundamental interplay between microscale and macroscale properties of human brain connectivity might form a fruitful new framework for the examination of dysconnectivity effects in neurological and psychiatric brain disorders because many are reported to involve affected connectivity on both ends of the organizational scale.

\section{Notes}

Supplemental material for this article (a description of Von Economo and Koskinas mapping to the Desikan-Killiany atlas) is available at www. dutchconnectomelab.org. This material has not been peer reviewed.

\section{References}

Amunts K, Zilles K (2012) Architecture and organizational principles of Broca's region. Trends Cogn Sci 16:418-426. CrossRef Medline

Barbas H (2015) General cortical and special prefrontal connections: principles from structure to function. Annu Rev Neurosci 38:269-289. CrossRef Medline

Barbas H, Rempel-Clower N (1997) Cortical structure predicts the pattern of corticocortical connections. Cereb Cortex 7:635-646. CrossRef Medline

Beul SF, Grant S, Hilgetag CC (2014) A predictive model of the cat cortical connectome based on cytoarchitecture and distance. Brain Struct Funct. In press.

Brodmann K (1909) Vergleichende Lokalisationslehre der Grosshirnrinde. Leipzig: Johann Ambrosius Barth.

Cammoun L, Gigandet X, Meskaldji D, Thiran JP, Sporns O, Do KQ, Maeder P, Meuli R, Hagmann P (2012) Mapping the human connectome at multiple scales with diffusion spectrum MRI. J Neurosci Methods 203: 386-397. Medline

Dehaene S, Naccache L (2001) Towards a cognitive neuroscience of consciousness: basic evidence and a workspace framework. Cognition 79: 1-37. CrossRef Medline

Dehaene S, Kerszberg M, Changeux JP (1998) A neuronal model of a global workspace in effortful cognitive tasks. Proc Natl Acad Sci U S A 95: 14529-14534. CrossRef Medline

de Reus MA, van den Heuvel MP (2014) Simulated rich club lesioning in brain networks: a scaffold for communication and integration? Front Hum Neurosci 8:647. Medline

Elston GN (2003) Cortex, cognition and the cell: new insights into the pyramidal neuron and prefrontal function. Cereb Cortex 13:1124-1138. CrossRef Medline

Elston GN, Benavides-Piccione R, DeFelipe J (2001) The pyramidal cell in cognition: a comparative study in human and monkey. J Neurosci 21 : RC163. Medline

Fischl B, van der Kouwe A, Destrieux C, Halgren E, Ségonne F, Salat DH, Busa E, Seidman LJ, Goldstein J, Kennedy D, Caviness V, Makris N, Rosen B, Dale AM (2004) Automatically parcellating the human cerebral cortex. Cereb Cortex 14:11-22. CrossRef Medline

Hagmann P, Cammoun L, Gigandet X, Meuli R, Honey CJ, Wedeen VJ, Sporns O (2008) Mapping the structural core of human cerebral cortex. PLoS Biol 6:e159. CrossRef Medline

Hilgetag CC, Grant S (2010) Cytoarchitectural differences are a key determinant of laminar projection origins in the visual cortex. Neuroimage 51:1006-1017. CrossRef Medline

Jacobs B, Driscoll L, Schall M (1997) Life-span dendritic and spine changes in areas 10 and 18 of human cortex: a quantitative Golgi study. J Comp Neurol 386:661-680. Medline

Jacobs B, Schall M, Prather M, Kapler E, Driscoll L, Baca S, Jacobs J, Ford K, Wainwright M, Treml M (2001) Regional dendritic and spine variation in human cerebral cortex: A quantitative golgi study. Cereb Cortex 11: 558-571. CrossRef Medline 
Jbabdi S, Johansen-Berg H (2011) Tractography: where do we go from here? Brain Connect 1:169-183. CrossRef Medline

Lichtman JW, Sanes JR (2008) Ome sweet ome: what can the genome tell us about the connectome? Curr Opin Neurobiol 18:346-353. CrossRef Medline

Markov NT, Ercsey-Ravasz M, Lamy C, Ribeiro Gomes AR, Magrou L, Misery P, Giroud P, Barone P, Dehay C, Toroczkai Z, Knoblauch K, Van Essen DC, Kennedy H (2013) The role of long-range connections on the specificity of the macaque interareal cortical network. Proc Natl Acad Sci U S A 110:5187-5192. CrossRef Medline

Mesulam MM (1998) From sensation to cognition. Brain 121:1013-1052. CrossRef Medline

Oh SW, Harris JA, Ng L, Winslow B, Cain N, Mihalas S, Wang Q, Lau C, Kuan L, Henry AM, Mortrud MT, Ouellette B, Nguyen TN, Sorensen SA, Slaughterbeck CR, Wakeman W, Li Y, Feng D, Ho A, Nicholas E, et al. (2014) A mesoscale connectome of the mouse brain. Nature 508:207214. CrossRef Medline

Sanides F (1970) Functional architecture of motor and sensory cortices in primates in the light of a new concept of neocortex evolution. The primate brain: advances in primatology. New York: Appleton.

Scholtens LH, Schmidt R, de Reus MA, van den Heuvel MP (2014) Linking macroscale graph analytical organization to microscale neuroarchitectonics in the macaque connectome. J Neurosci 34:12192-12205. CrossRef Medline

Scholtens LH, de Reus MA, van den Heuvel MP (2015) Linking contemporary high-resolution magnetic resonance imaging to the von Economo legacy: a study on the comparison of MRI cortical thickness and histological measurements of cortical structure. Hum Brain Mapp 36:3038-3046. CrossRef Medline

Schuz A (2002) Cortical areas: unity and diversity. London: Taylor and Francis.

Triarhou LC (2008) Atlas of Cytoarchitectonics of the Adult Human Cor- tex; Translated and edited version of 'Die cytoarchitektonik der hirnrinde des erwachsenen menschen' (Von Economo and Koskinas, 1925). KARGER.

Triarhou LC (2009) Cellular Structure of the Human Cerebral Cortex; Translated and edited version of 'Zellaufbau der Grosshirnrinde des Menschen' (von Economo, 1927). Basel: Karger.

van den Heuvel MP, Sporns O (2011) Rich-club organization of the human connectome. J Neurosci 31:15775-15786. CrossRef Medline

van den Heuvel MP, Sporns O (2013) Network hubs in the human brain. Trends Cogn Sci 17:683-696. CrossRef Medline

van den Heuvel MP, Kahn RS, Goñi J, Sporns O (2012) High-cost, highcapacity backbone for global brain communication. Proc Natl Acad Sci U S A 109:11372-11377. CrossRef Medline

Van Essen DC, Smith SM, Barch DM, Behrens TE, Yacoub E, Ugurbil K; WU-Minn HCP Consortium (2013) The WU-Minn Human Connectome Project: an overview. Neuroimage 80:62-79. CrossRef Medline

Vogt O, Vogt C (1903) Zur anatomischen Gliederung des Cortex cerebri. J Psychol Neurol 2:160-180.

Von Economo CF (1927) Zellaufbau der Grosshirnrinde des Menschen. Berlin: Springer.

Von Economo CF, Koskinas GN (1925) Die Cytoarchitektonik der Hirnrinde des erwachsenen Menschen. Berlin: Springer.

Yuste R (2011) Dendritic spines and distributed circuits. Neuron 71:772781. CrossRef Medline

Zilles K, Palomero-Gallagher N, Bludau S, Mohlberg H, Amunts K (2015a) Cytoarchitecture and maps of the human cerebral cortex. In: Brain mapping: an encyclopedic reference (Toga AW, ed.), pp 115-135. San Diego: Elsevier Academic.

Zilles K, Palomero-Gallagher N, Amunts K (2015b) Myeloarchitecture and maps of the cerebral cortex. In: Brain mapping: an encyclopedic reference (Toga AW, ed.), pp 137-156. San Diego: Elsevier Academic. 